\title{
La lutte par piégeage contre Glossina fuscipes fuscipes pour la protection de l'élevage en République centrafricaine. J.P. Gouteux 'II. Caractéristiques du piège bipyramidal
}

\begin{abstract}
GOUTEUX (J.P.). La lutte par piégeage contre Glossina fuscipes fuscipes pour la protection de l'élevage en République centrafricaine. II. Caractéristiques du piège bipyramidal. Revue Élev. Méd. vét. Pays trop., 1991, 44 (3) : 295-299

L'auteur donne la description et le mode de fabrication du piège bipyramidal. Ce piège fait actuellement l'objet d'un programme d'évaluation en vraie grandeur dans le cadre d'une lutte anti-tsé-tsé autogérée par les éleveurs semi-nomades de République centrafricaine (Mbororo). Il est démontable, facilement transportable et ne demande aucune manipulation complexe. Le prix de revient d'un piège est d'environ $30 \mathrm{FF}$ au maximum (1 500 francs CFA). Mots clés: Glossina fuscipes fuscipes - Lutte anti-insecte - Piège bipyramidal Protection de l'élevage - Éleveur Peul - République centrafricaine.
\end{abstract}

\section{INTRODUCTION}

Un des premiers impératifs de mise en place d'une lutte anti-tsé-tsé autogérée par les éleveurs est de disposer d'un piège au coût relativement bas, compatible avec le budget moyen d'un éleveur centrafricain et pouvant être compétitif avec le coût des traitements préventifs et curatifs des trypanosomoses (3).

Les dépenses familiales moyennes en produits vétérinaires s'élèvaient en 1990 à 36600 francs CFA par an (6). Une enquête auprès de la Fédération Nationale des Éleveurs Centrafricains (FNEC) montre que les trypanocydes (Bérénil ${ }^{R *}$, Trypamidium ${ }^{\text {} * \star}$, Novidium ${ }^{R \star \star \star}$ ) représentent 65 à 75 p. 100 des chiffres globaux de vente de ces produits (2). Le montant consacré à la lutte contre les trypanosomoses est donc de 23 à 27000 francs CFA. Le piège préalablement utilisé en République centrafricaine était le piège biconique (1) dont le prix se situait en 1990

\section{ORSTOM, BP 893, Bangui, République centrafricaine.}

Reçu le 17.12.1990, accepté le 17.6.1991.

* Diminazène. ${ }^{* *}$ Chlorhydrate de chlorure d'isométamidium. *** Chlorure d'homidium.

**** Ce dernier prix n'étant obtenu que grâce à la fabrication dans un atelier de la FNEC des parties métalliques du piège (piquet, cage, support apicale, arceau) et étant encore volontairement sous-estimé.

***** Pyréthrinoïde de synthèse commercialisé sous le nom de $\mathrm{K}$ Othrine ${ }^{R}$ par Roussel-Uclaf (C.E. à $25 \mathrm{~g} / \mathrm{l}$ ). dans une fourchette de 7500 à 15000 Francs CFA, ce qui est très lourd pour le budget d'un éleveur (LE MASSON, comm. pers.). De fait, aucun exemplaire de ce piège commercialisé en 1990 par la FNEC au prix de 7500 francs CFA**** n'a été vendu.

Le piège bipyramidal est un nouveau piège conçu dans le cadre d'un programme de recherche de l'Agence Nationale de Développement de l'Élevage (ANDE) sur la protection de l'élevage en zone de savane humide. $\mathrm{Ce}$ piège a été mis au point dans un but d'autogestion de la lutte par les éleveurs, en tenant compte des contraines imposées par cette stratégie. Les essais ayant permis sa conception font l'objet de la première partie de cet article (5). La présente partie en donne le mode de fabrication (façon, matériaux), la description, le fonctionnement et enfin une étude détaillée du coût.

\section{DESCRIPTION}

\section{Corps du piège}

Ce piège (photo 1) n'a ni arceau ni cône métalliques. Sa forme est donnée par trois baguettes de bois d'un mètre environ. Ces baguettes sont amovibles, maintenues par un gousset de tissu aux deux extrémités. Le piège est suspendu, il ne nécessite donc pas de piquet de métal. II est pliable (il tient dans une enveloppe $21 \times 29,7 \mathrm{~cm}$ ) et donc facilement transportable.

\section{Système de capture}

Le système de capture choisi après des essais comparatifs (5) est composé d'une bouteille de plastique souple (en polyéthilène de haute densité PEHD) enfichée sur un cône de bouteille découpée. Celui-ci fait fonction de dispositif anti-retour et de point d'attache d'une cordelette qui servira à la suspension du piège. Le système se vide par le goulot fermé par un bouchon. Une solution d'insecticide passée dans la bouteille (deltaméthrine ${ }^{\star \star \star \star \star}$ diluée à $10 \mathrm{mg} / \mathrm{l}$ ) est suffisante pour imprégner les parois de façon durable. Ceci présente surtout l'intérêt d'empêcher la prédation des glossines tuées par les fourmis, car les mouches capturées ne survivent pas longtemps à l'insolation à l'intérieur de la bouteille (effet de serre). 


\section{J.P. Gouteux}

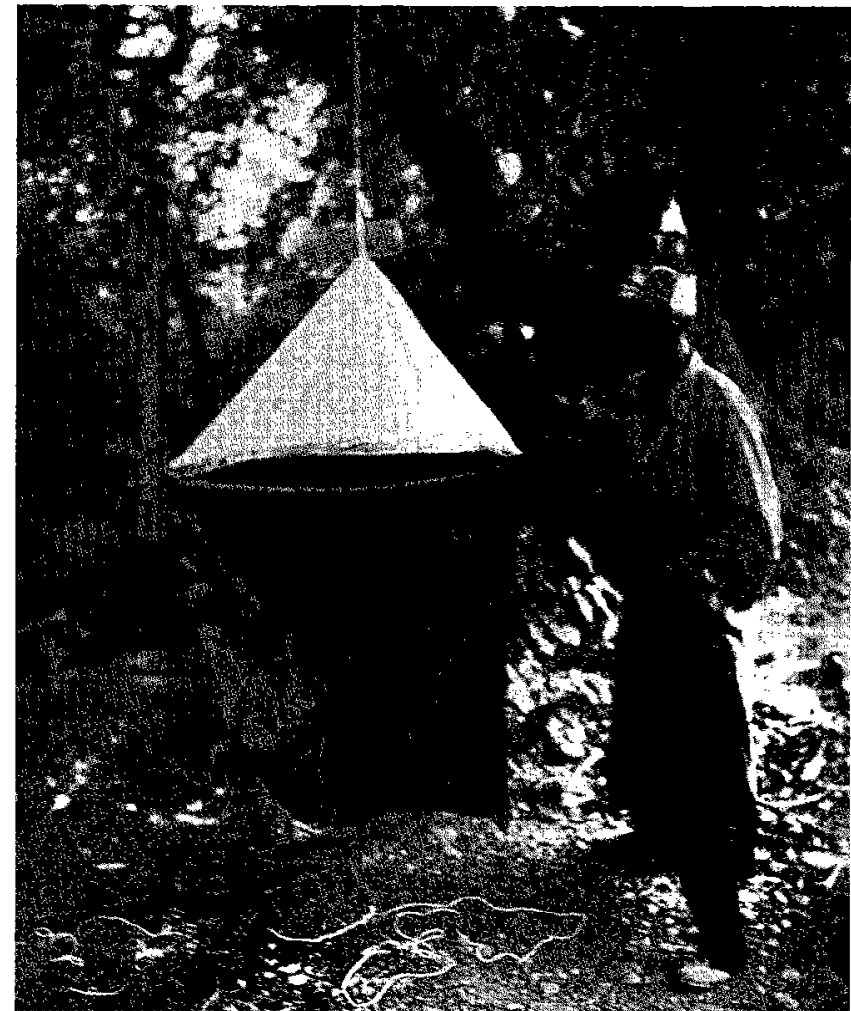

Photo 1 : Piège bipyramidal en place près d' un abreuvoir.

Ce système est paradoxalement à la fois le plus efficace, puisqu'un piège équipé de ce système a un rendement équivalent à deux ou trois pièges identiques équipés de cages Roubaud, et le moins cher. II ne coûte que $125 \mathrm{~F}$ CFA contre plus de 2500 F CFA pour la cage Roubaud et son support.

\section{FABRICATION}

\section{Matériaux}

Le tulle moustiquaire utilisé (Ondine, fournisseur : Tarare, France, réf. 1121 AA maille DT6) est vendu sous forme de rouleaux de $30,4 \mathrm{~m}$ de long et $1,2 \mathrm{~m}$ de large. II est à 100 p. 100 en polyester avec une maille de 2,5 mm.

Les films plastique (fabricant : Plastherm, Montreuil, France, réf. films PE Laize $n^{\circ} 4021$ bleu et $n^{\circ} 1821$ noir) sont en polyéthylène et présentés sous forme de rouleaux de $1,03 \mathrm{~m}$ de large pesant $100 \mathrm{~kg}$, soit l'équivalent d'un peu plus de $1000 \mathrm{~m}$ par rouleau. La coloration du

film bleu est donnée de façon objective par sa courbe de réflectivité (5). Les dẹu films ont été mis au point spécialement par la firme pour faire des pièges à tsé-tsé, utilisés à l'origine en Ouganda (LANCIEN, comm. pers.). Ces deux produits sont caractérisés, selon cette firme, "par une résistance de $300^{\circ} \mathrm{C}$ à la chaleur, de 8 à la lumière" et qualifiée d'"excellente" à la migration (Plastherm, Comm. pers., 19.10.1990). Le pourcentage d'opacité du bleu est environ de 10 p. 100 , celui du noir de 8 p. 100. Leur durée de vie dans des conditions d'ensoleillement maximal ainsi que les modifications de la coloration sont en cours d'étude. Ils sont garantis par le fabricant pour une durée de vie de 16 mois à deux ans.

\section{Coupe}

La coupe des matériaux de ce piège, tulle et plastique, a été conçue de façon à ce que la fabrication du piège ne laisse aucune perte : il n'y a pas de chute. Pour un piège, il faut : $0,90 \mathrm{~m}$ de plastique noir (en 1,00 $\mathrm{m}$ de large) ; $2,25 \mathrm{~m}$ de plastique bleu (en 1,00 $\mathrm{m}$ de large) ; $0,75 \mathrm{~m}$ de tulle moustiquaire (en 1,20 $\mathrm{m}$ de large).

Le détail de la coupe de ces matériaux est donné en figure 1. Un rouleau de tulle permet la confection de 40 pièges, un rouleau de plastique plus de 450 et 1000 pièges, respectivement pour le bleu et le noir.

A

$\stackrel{0,6 \mathrm{~m}}{\stackrel{1}{ }}$

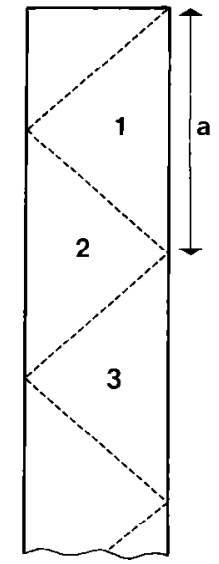

B

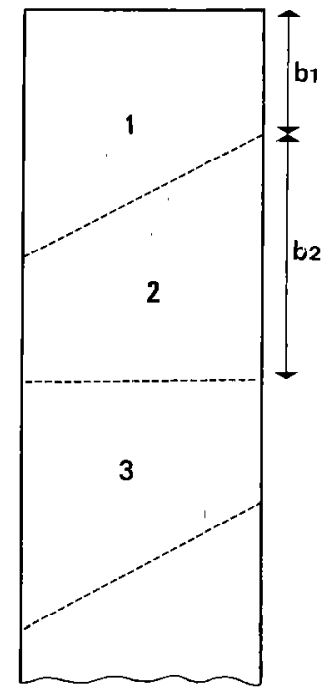

Fig. I : Schéma de découpe des matériaux. A : tulle moustiquaire en $1 / 2$ largeur $(a=l \mathrm{~m}) ; B$ : plastique bleu $(b 1=0,5 \mathrm{~m}, b 2=1 \mathrm{~m}) ; C$ : plastiquc noir $(c l=0,6 m, c 2=1,2 \mathrm{~m})$. Les tirets indiquent les lignes de découpe. $I l$ faut trois parties (numérotées de 1 à 3 ) de chaque matériau pour faire un piège (voir fig. 2). 


\section{Assemblage}

L'assemblage des trois parties du piège se fait simultanément (fig. 2). Une marge de 1 à $2 \mathrm{~cm}$ est réservée pour les ourlets. L'ouverture du piège est ensuite découpée selon un gabarit. Les goussets sont réalisés comme indiqué sur la figure 3 . Un tailleur, avec un aide

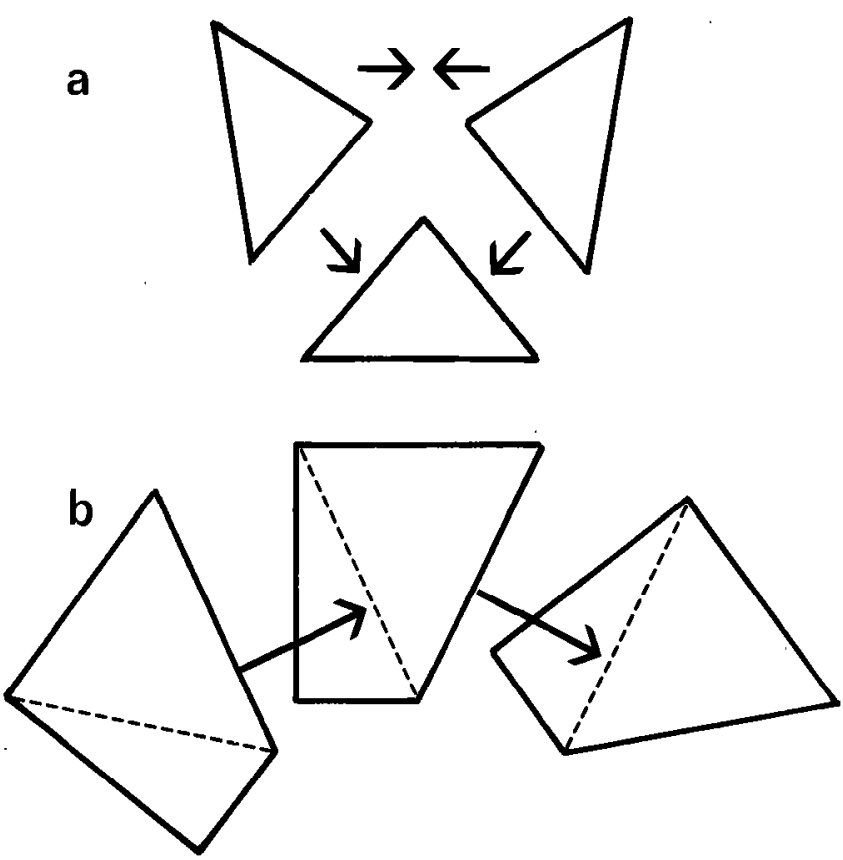

C

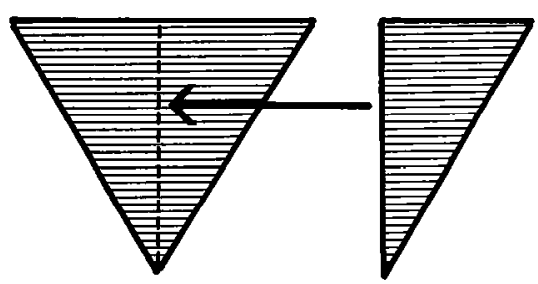

d

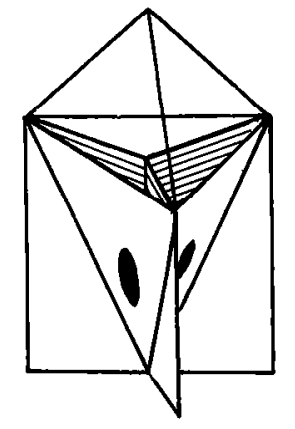

Fig. 2 : Assemblage du piège. $a$ : pyramide supéricure en tulle $; b$ : pyramide inférieure bleue; $c$ : écrans inférieurs noirs; $d$ : piège composé des parties $a+b+c$. Dans la pratique, l'assemblage de ces trois parties se fait simultanément. Les tirets indiquent les lignes d'assemblage.

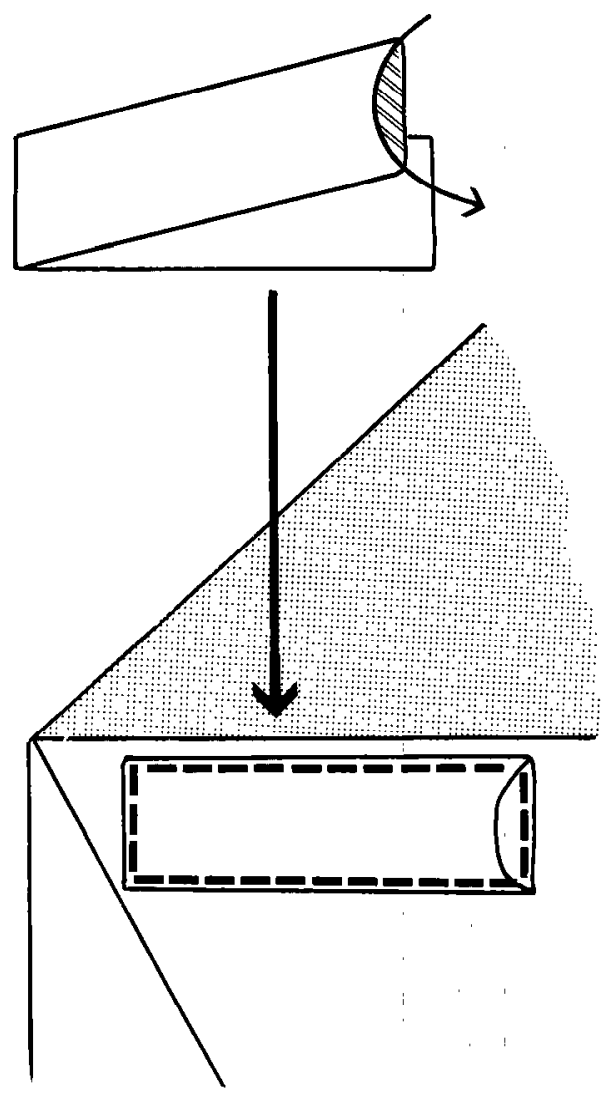

Fig. 3 : Schéma de découpe et de montage du gousset de tissu pour la fixation des baguettes.

pour la découpe des matériaux, assemble facilement 20 à 25 pièges par jour. II est recommandé de percer le bas du piège pour éviter l'accumulation d'eau de pluie.

\section{FONCTIONNEMENT}

\section{Montage}

L'éleveur se procure trois baguettes souples d'environ un mètre (les pétioles émincés de palmiers font parfaitement l'affaire). Chaque baguette est introduite dans un gousset et pliée pour pénétrer le deuxième gousset. Un petit cordon de tissu est fixé au milieu de chaque côté et, s'attachant à la baguette, permet un bon déploiement du piège. Celui-ci est suspendu par le système de capture, à proximité de l'abreuvoir (près des berges), à l'aide de cordes artisanales (ou lianes), dans un endroit aussi visible que possible, de préférence ensoleillé, surtout en saison des pluies. 


\section{J.P. Gouteux}

\section{Entretien}

Il est impératif de surveiller la montée des eaux pour reculer le piège des berges en période de crue. Les déchirures sont réparées avec du fil à coudre. Le piège ne nécessite aucune opération manuelle particulière, les tsé-tsé prises étant tuées par le système de capture. II est possible de compter les mouches périodiquement ou lors de l'évacuation du trop-plein des captures.

\section{Matériaux}

Tulle moustiquaire : le prix d'achat hors taxes du mètre en France est de 5,60 FF. Le prix d'achat rendu à Bangui incluant tous les frais annexes (transport, transitaire) est d'environ $8 \mathrm{FF} / \mathrm{m}(400 \mathrm{~F} \mathrm{CFA} / \mathrm{m})$, soit pour un piège $6 \mathrm{FF}$.

Plastique bleu et noir : le prix d'achat hors taxes du $\mathrm{kg}$ en France est de $26,50 \mathrm{FF}$ ( $1 \mathrm{~kg}=12,5 \mathrm{~m}$ environ), soit un prix de revient à Bangui, incluant tous les frais annexes, d'environ $3 \mathrm{FF} / \mathrm{m}$ (150 F CFA), soit pour un piège environ 3 FF pour le noir et 7 FF pour le bleu.

Autres matériaux et accessoires : le prix des autres matériaux achetés sur place, pour la fabrication d'un piège, est le suivant : K-Othrine 0,16 FF, ficelle 0,34 FF, bouteilles $2,00 \mathrm{FF}$, fil à coudre $0,10 \mathrm{FF}$, soit un total de 2,60 FF.

Le coût de la totalité des matériaux d'un piège est donc de 18,60 FF, soit environ 20 FF ou 1000 F CFA.

\section{Fabrication}

Le prix payé par unité au tailleur est de $6 \mathrm{FF}$, auquel on peut ajouter comme frais annexes et participations diverses $4 \mathrm{FF}$, ce qui fait un total de $10 \mathrm{FF}$ par piege.

Le coût* total d'un piège est donc de $30 \mathrm{FF}$ ou 1500 francs CFA.

GOUTEUX (J.P.). Control of Glossina fuscipes fuscipes by traps to protect livestock in the Central African Republic. II. Characteristics of the bipyramidal trap. Revue Élev. Méd. vét. Pays trop., 1991, 44 (3) : 295-299

The author describes the properties and manufacturing of a bipyramid trap. Building details are provided. It is easy to handle, settle and dismantle for transportation and does not need any special preparaliun such as an insecticidal impregnation. Its cost price is about $30 \mathrm{FF}$ (1 500 CFA francs). The use of this trap by semi-nomadic breeders is actually submitted to a large scale evaluation programme. Key words: Glossina fuscipes fuscipes - Inscet control - Bipyramidal trap - Livestock protection - Peul breeder - Central African Republic.

\section{DISCUSSION}

Ce prix peut être facilement compressé par la récupération des bouteilles, l'abaissement du prix de la confection à $4 \mathrm{FF}$ en fonction de la production, la suppression de divers frais évitables, l'achat en plus grande quantité de matériaux, d'où la possibilité d'un coût total pour un piège inférieur à $24 \mathrm{FF}$ (1 $200 \mathrm{~F} \mathrm{CFA}$ ).

\section{CONCLUSION}

Le piège bipyramidal améliore de dix fois le meilleur rapport efficacité/coût du piège biconique. II fait actuellement l'objet d'une évaluation en vraie grandeur dans le cadre d'une lutte autogérée par les éleveurs semi-nomades de République centrafricaine (Mbororo). La stratégie d'utilisation de ce piège ne vise pas ici une éradication des tsétsé, même localisée, mais un allègement de la pression vectorielle dans les points de contact tsé-tsé/bétail : les portions de galeries défrichées par les éleveurs pour abreuver leurs bêtes. Son utilisation dans d'autres contextes et à d'autres fins est envisageable : protection de ranch, lutte intégrée, etc. En Centrafrique, c'est la même espèce de tsé-tsé, G. fuscipes fuscipes Newstead, 1910 , qui est le principal vecteur des trypanosomoses animales dans les zones d'élevage et de la maladie du sommeil à $T$. brucei gambiense, Dutton, 1902, dans le plus grand foyer du pays (Nola, en zone forestière). Conçu à l'origine pour un programme de recherche sur la protection de l'élevage, l'utilisation de ce piège dans le cadre de la santé humaine pourra donc être une heureuse retombée.

* Le détail précis des calculs des coûts est donné dans un rapport disponible sur demande à l'auteur (4). Le piège bipyramidal est actuellement commercialisé par la FNEC à $2000 \mathrm{~F}$ CFA dans son réseau de vente et à 1750 F CFA pour les Groupements d'Intérêt Pastoraux (GIP).

GOUTEUX (J.P.). La lucha con trampas contra Glossina fuscipes fuscipes para la protección de la ganadería en República Centroafricana. II. Características de la trampa bipiramidal. Revue Élev. Méd. vét. Pays trop., 1991, 44 (3) : 295-299

El autor describe la trampa bipiramidal y el modo de fabricación. Actualmente se desarrolla un programa de evaluación de la trampa con arreglo a una lucha contra las moscas tse-tsé automanejada por los ganaderos seminómados de República Centroafricana (Mbororo). Es desmontable, facilmente transportable y sin manejo complejo. Es de unos 30 FF al maximo ( 1500 F CF ) el precio de coste de una trampa. Palabras claves : Glossina fuscipes fuscipes - Lucha contra los insectos - Trampa bipiramidal - Protección de la ganadería - Ganadero Peul - República Centroafricana. 


\section{BIBLIOGRAPHIE}

1. CHALLIER (A.), EYRAUD (M.), LAFAYE (A.), LAVEISSIERE (C.). Amélioration du rendement du piège biconique pour les glossines (Diptera, Glossinidae) par l'emploi d'un cône inférieur bleu. Cah. ORSTOM, Sér. Ent. méd. Parasit., 1977, 15 : 283-286.

2. CUISANCE (D.). Bilan de quatre missions d'appui à l'unité de lutte contre les glossines dans le cadre du projet national de développement de l'élevage. Maisons-Alfort, Rapport IEMVT-BDPA-SCETAGRI, $1988.62 \mathrm{p}$.

3. CUISANCE (D.), GOUTEUX (J.P.), CAILTON (P.), KOTA-GUINZA (A.), N'DOKOUE (F.), POUNEKROUZOU (E.), DEMBA (D.). Problématique d'une lutte contre les glossines pour la protection de l'élevage zébu en République centrafricaine. Soc. $r$. belge Ent., 1991, 35 (sous presse).

4. GOUTEUX (J.P.), BLANC (F.). Coût du piège bipyramidal à tsé-tsé "ANDE”. Bangui, ORSTOM-ANDE, 1990. 3 p.

5. GOUTEUX (J.P.), CUISANCE (D.), DEMBA (D.), N'DOKOUE (F.), LE GALL (F.). La lutte par piégeage contre Glossina fuscipes fuscipes pour la protcction de l'ćlevage en République centrafricainc. I. Misc au point d'un piège adapté à un milieu d'éleveurs semi-nomades. Revue Élev. Méd. vét. Pays trop., 1991, 44 (3) : 287-294.

6. LE MASSON (C.), REMAYEKO (A.). Les éleveurs Mbororo. Étude socio-économique. Rangui, ANDE, 1990. 227 p. 